\title{
Estimation of fracture compliance from tubewaves generated at a fracture intersecting a borehole
}

Sudhish K. Bakku*, Michael Fehler and Daniel R. Burns, Earth Resource Laboratory, MIT

\section{SUMMARY}

Understanding fracture compliance is important for characterizing fracture networks and for inferring fluid flow in the subsurface. In an attempt to estimate fracture compliance in the field, we developed a new model to understand tubewave generation at a fracture intersecting a borehole. Solving the dispersion relation in the fracture, amplitude ratios of generated tubewave to incident P-wave were studied over all frequency ranges. Based on the observations from the model, we propose that measuring amplitude ratios near a transition frequency can help constrain fracture compliance and aperture. The transition frequency corresponds to the regime where the viscous skin depth in the fracture is comparable to its aperture. However, measurements in the high frequency limit can place a lower bound on fracture compliance. Comparing the model to a previously published VSP dataset, we argue that compliance values of the order $10^{-10}-10^{-9} \mathrm{~m} / \mathrm{Pa}$ may be possible in the field.

\section{INTRODUCTION}

Fracture networks account for a significant amount of flow in many petroleum reservoirs, especially in carbonate reservoirs and other less porous formations. Considerable research has been done to characterize fractures through effective medium theories (Peacock and Hudson, 1990; Kachanov, 1992). It is common to apply methods like amplitude variation with offset and angle (AVOA) to characterize the velocity anisotropy which can be interpreted to characterize preferred orientation of the fractures and anisotropy in permeability. However, the focus is increasingly on detecting larger discrete fractures that are connected over reservoir scales and that are more conducive to fluid flow. These macrofractures have lateral extent comparable to the wavelength of the incident wavefield (tens of meters) and have separations on the order of a wavelength. Such fractures can scatter the seismic wavefield. Several authors developed methods to analyze scattered coda to estimate fracture orientation and spacing (Willis et al., 2006; Burns et al., 2007; Grandi, 2008). These methods are based on the results from finite difference models that required large compliance values (of the order of $10^{-10} \mathrm{~m} / \mathrm{Pa}$ ) to generate scattered waves with significant amplitude to be observed. Fractures are implemented in the finite difference scheme as linearslip boundaries (Schoenberg, 1980) by giving the nodes corresponding to the fracture locations an excess compliance in addition to the background compliance. However, the range of compliance values that natural fractures admit are not clear.

Previous laboratory experiments on single fractures in core samples, at ultra-sonic frequencies, suggested a range of compliance values in between $10^{-14}-10^{-13} \mathrm{~m} / \mathrm{Pa}$ (Pyrak-Nolte et al., 1990; Lubbe et al., 2008). However, cross-hole studies in the field, at $\mathrm{kHz}$ frequencies, placed the compliance estimates at $2.5 \times 10^{-13}-2 \times 10^{-12} \mathrm{~m} / \mathrm{Pa}$ (King et al., 1986; Lubbe and Worthington, 2006). Worthington and Hudson (2000) studied attenuation of VSP signals across a fault and roughly estimated the compliance of the fault to be of the order of $10^{-9} \mathrm{~m} / \mathrm{Pa}$ or greater. In all these studies, the dimension of the fracture and the probe frequency varied over orders of magnitude. Based on this, Worthington and Lubbe (2007) argued that fracture compliance may scale with the size of the fracture. However, the data are too limited to apply this rule. It is essential to measure fracture compliance in-situ at exploration frequencies.

In this paper, we describe a method to estimate the compliance of fractures intersecting a borehole, from VSP data. Previously, Hardin et al. (1987) developed a similar model to estimate fracture compliance. However, his model is limited to the low-frequency regime, when the viscous skin depth in the fracture is much larger than the fracture aperture.

\section{THEORY}

We consider a horizontal fracture, infinite in lateral extent and intersecting a vertical borehole of radius $R$ (see Figure 1). A model is developed to study the tubewave generation in the borehole when a plane $\mathrm{P}$-wave is normally incident on the fracture. However, the results can be extended to an arbitrary angle of incidence. When the wavefield is incident on the fluid-filled fracture, it squeezes and expels the fluid into the borehole, generating a tubewave. The amplitude of the tubewave is proportional to the amount of fluid exchanged between the fracture and the borehole. The fluid exchange, in turn, depends on the compliance and fluid transmissivity of the fracture.

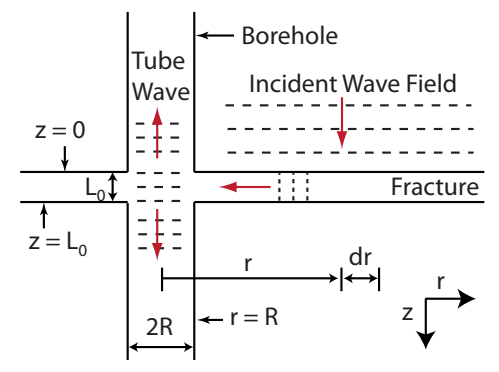

Figure 1: Cartoon showing tubewave generation at a fracture intersecting a borehole.

Fractures are discontinuities in the subsurface, held open by asperities that resist the fracture closure. For simplicity, we assume the fracture to be a parallel plate with static aperture, $L_{0}$, and compliance, $Z$. Here, we neglect the effect of roughness, tortuosity and actual contact area of fracture on the fluid motion in the fracture. Fracture closure is proportional to the compliance and the applied effective normal stress. Incorporating this, dynamic fracture aperture at any location can be written as (Hardin et al., 1987) 


\section{Fracture compliance from tubewaves}

$$
L(t)=L_{0}+Z\left[p(t)-\sigma_{n}(t)\right]
$$

where $\sigma_{n}(t)$ is the normal stress on the fracture face, and $p(t)$ is the perturbation in the fracture fluid pressure due to closure. For a normally incident $\mathrm{P}$-wave, $\sigma_{n}(t)=\sigma_{0} e^{-i \omega t}$, where $\sigma_{0}, \omega$ are the stress amplitude and frequency of the incident wave. The incident wave perturbs the fracture aperture and causes a pressure gradient in the fracture. For simplicity, fluid pressure and flow in the fracture are averaged over the aperture and only their radial variation is considered. Due to the axial symmetry of the problem, we use cylindrical co-ordinates (see Figure 1). The net flow out of a volume element, $2 \pi r L(t) d r$, between $r$ and $r+d r$ from the axis of the borehole, during a time increment $d t$, should equal the change in volume of the element, during the same time, due to perturbation in the aperture and the change in the fluid volume due to compressibility of the fluid. Thus, we arrive at

$$
-\left(\frac{\partial q}{\partial r}+\frac{q}{r}\right)=\frac{d L}{d t}+L \gamma \frac{\partial p}{\partial t}
$$

where $\gamma$ is the fluid compressibility and $q$ is the radial flow per unit length. Flow in the above equation can be related to the pressure gradient through dynamic conductivity, $\bar{C}$. Solving for the flow field in a rigid fracture, Tang (1990) showed that the flow averaged over the aperture at any location can be related to the radial pressure gradient at that location as

$$
\bar{q}=-\bar{C} \frac{\partial \bar{p}}{\partial r}, \text { where } \bar{C}=\frac{i \omega L_{0}}{k_{r}^{2} \alpha_{f}^{2} \rho_{f}}
$$

where $\alpha_{f}$ is the acoustic velocity in the fluid, $\rho_{f}$ is the fluid density and $i$ is the imaginary unit. The bar over the symbols denotes that the quantities are in the frequency domain. $k_{r}$ is the radial wavenumber of those specific modes that can exist in the fracture and is obtained by solving the dispersion relation for the velocity field in the fracture (Tang, 1990). After neglecting higher order terms, using equations 1,2 and 3 , we can write the differential equation for fluid pressure in the fracture in the frequency domain as

$$
\frac{\partial^{2} \bar{p}}{\partial r^{2}}+\frac{\partial \bar{p}}{\partial r}+\frac{k_{r}^{2} \alpha_{f}^{2}}{\alpha_{e f f}^{2}} \bar{p}=\frac{\rho_{f} Z k_{r}^{2} \alpha_{f}^{2}}{L_{0}} \sigma_{0}
$$

where $\alpha_{e f f}^{2}=\frac{1}{\rho_{f}} \frac{1}{\left(\gamma+\frac{Z}{L_{0}}\right)}$, with boundary conditions:

$\bar{p}(r=R, \omega)=\bar{p}_{t}(\omega)$, where $\bar{p}_{t}(\omega)$ is the pressure due to the tubewave generated in the borehole. As $r \rightarrow \infty$, fluid motion in the radial direction tends to zero and we require $\frac{\partial \bar{p}}{\partial r}=0$. However, Tang (1990) showed that viscous or inertial forces dominate depending on the magnitude of viscous skin depth, $\delta=\sqrt{\frac{2 v}{\omega}}$, relative to the fracture aperture, $L_{0}$, where, $v$ is the kinematic viscosity of the fluid. Under the high frequency approximation $\left(\frac{\delta}{L_{0}} \ll 1\right), k_{r}^{2}$ is a real number and the differential equation for pressure takes the form of a wave equation as the inertial forces dominate. Under the low frequency approximation $\left(\frac{\delta}{L_{0}} \gg 1\right), k_{r}^{2}$ is imaginary and the pressure follows a diffusion equation as the viscous forces dominate. For the frequencies of interest (10-100s of Hz) and for the range of natural fracture apertures $(0.1-0.5 \mathrm{~mm}), \frac{\delta}{L_{0}}$ is close to unity and it is not clear if high or low frequency approximation is valid. In this transition zone, $k_{r}^{2}$ is complex and pressure has both wave and diffusive components. To address this, for an arbitrary frequency, we solved the dispersion relation numerically for $k_{r}$. The dispersion relation has multiple solutions. However, we observe that the contribution of higher modes to flow is negligible compared to that of fundamental mode and it is sufficient to consider just the fundamental mode. The fundamental mode converged to the solutions predicted by Tang (1990) at high and low frequency limits. Taking this numerical solution for $k_{r}$, equation 4 encapsulates both diffusion and propagation. The homogenous solutions to equation 4 are Hankel functions of first and second kind taking complex arguments. However, since the fundamental mode lies in the upper half of the complex plane, the Hankel function of the second kind goes to infinity as $r \rightarrow \infty$, while the first kind approaches zero, satisfying the boundary condition. Taking Hankel functions of the first kind and satisfying the boundary condition at the borehole, the pressure in the fracture can be written as

$$
\bar{p}(\omega, r)=\left[\bar{p}_{t}(\omega)-\frac{\rho_{f} Z \alpha_{e f f}^{2}}{L_{0}} \sigma_{0}\right] \frac{H_{0}^{1}(\zeta r)}{H_{0}^{1}(\zeta R)}+\frac{\rho_{f} Z \alpha_{e f f}^{2}}{L_{0}} \sigma_{0}
$$

where, $\zeta=\frac{k_{r} \alpha_{f}}{\alpha_{e f f}}$ and $H_{m}^{n}$ is the Hankel function of the nth kind and order $\mathrm{m}$. Knowing the distribution of pressure in the fracture from the above equation, the pressure gradient at the borehole wall, $r=R$, can be found. The rate of volume injection into the borehole $\frac{d V}{d t}$ can be then estimated from equation 3 as

$$
\frac{d V}{d t}=-\left.2 \pi R \bar{q}\right|_{r=R}
$$

Since $\bar{q}$ denotes fluid flowing away from the origin, the negative sign is needed for fluid flow into the borehole. This fluid exchange between the fracture and the borehole, acts as a volume source and generates a tubewave of amplitude $p_{t}$ given by (Lee and Balch, 1982)

$$
p_{t}=\frac{\rho_{f} c_{t}}{2 \pi R^{2}} \frac{d V}{d t}
$$

where $c_{t}=\alpha_{f} / \sqrt{1+\rho_{f} \alpha_{f}^{2} /\left(\rho_{s} \beta^{2}\right)}$, is the tube-wave velocity, $\rho_{s}, \beta$ are the density and shear wave velocity of the formation, respectively. Thus, eliminating the rate of fluid injection from equations 6 and 7, we arrive at the expression for an equivalent pressure source for the tubewave in the frequency domain as

$$
\begin{aligned}
\bar{p}_{t}(\omega)= & \sigma_{0} \frac{\omega}{k_{r} \alpha_{f}} \frac{c_{t}}{\alpha_{e f f}} \frac{L_{0}}{R} \frac{\rho_{f} Z \alpha_{e f f}^{2}}{L_{0}} \times \\
& {\left[\frac{i H_{1}^{1}(\zeta R) / H_{0}^{1}(\zeta R)}{1+\frac{\omega}{k_{r} \alpha_{f}} \frac{c_{t}}{\alpha_{e f f}} \frac{L_{0}}{R} i H_{1}^{1}(\zeta R) / H_{0}^{1}(\zeta R)}\right] }
\end{aligned}
$$


Fracture compliance from tubewaves

At the same time, the incident $\mathrm{P}$-wave traveling along the borehole induces dynamic pressure in the borehole given by (White, 1983)

$$
\bar{p}_{i}(\omega)=\frac{\rho_{f} c_{t}^{2}}{\rho_{s} \beta^{2}}\left(\frac{1-2 \beta^{2} / \alpha^{2}}{1-c_{t}^{2} / \alpha^{2}}\right) \sigma_{0}
$$

By taking the ratio of $\bar{p}_{t}$ to $\bar{p}_{i}$, the amplitude ratio, we get rid of $\sigma_{0}$. The amplitude ratio can be easily determined from the power spectra of the VSP data and is indicative of fracture properties. Figure 2 shows the amplitude ratio plotted against frequency for a given fracture compliance and aperture. For comparison, the amplitude ratio found using the low and high frequency approximations are plotted as well. At the low frequency limit, the amplitude ratio tends towards zero (Ionov, 2007). With increasing frequency, the amplitude ratio increases and reaches a maximum at the transition from low to high frequency and then decreases with further increase in frequency. However, at high frequency, the amplitude ratio reaches a constant value. For a given compliance, the location of the peak in the amplitude ratio depends on the viscosity of the fluid and the fracture aperture. Increasing viscosity pushes the peak towards higher frequencies and larger aperture moves the peak towards lower frequencies. In general, as compliance increases, amplitude ratio increases over the entire frequency band (see Figure 3 ). Thus, amplitude ratios can be indicative of fracture compliance.

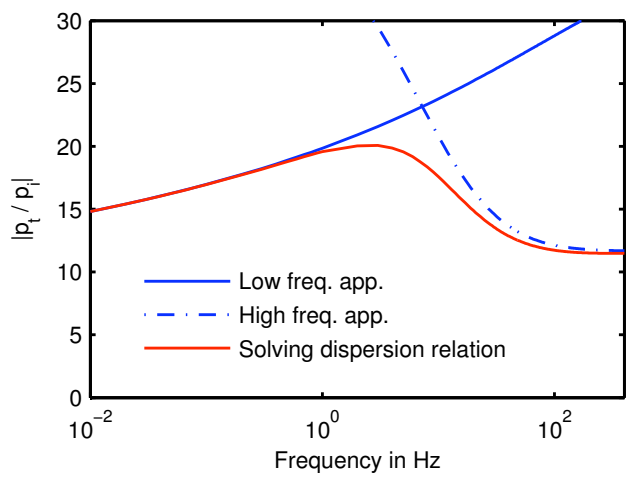

Figure 2: Tube to $\mathrm{P}$-wave pressure amplitude ratios are plotted against frequency assuming a fracture compliance of $10^{-9} \mathrm{~Pa} / \mathrm{m}$. The parameters for this study were $2 \mathrm{R}=15$ $\mathrm{cm}, L_{0}=0.55 \mathrm{~mm}, \alpha_{f}=1500 \mathrm{~m} / \mathrm{s}, \alpha=5800 \mathrm{~m} / \mathrm{s}, \beta=3300 \mathrm{~m} / \mathrm{s}$, $\rho_{f}=1000 \mathrm{~kg} / \mathrm{m}^{3}, \rho_{s}=2700 \mathrm{~kg} / \mathrm{m}^{3}, v=10^{-6} \mathrm{~m}^{2} / \mathrm{s}$. The fluid properties correspond to water.

\section{ESTIMATION OF FRACTURE COMPLIANCE}

In the field, we have a good knowledge of the formation velocities, fluid properties and the borehole diameter. The parameters that we know least about are the fracture compliance and the fracture aperture. Investigation of the behavior of the amplitude ratio as a function of compliance and aperture will allow us to use measured amplitude ratio to determine the two key fracture properties. We varied aperture from

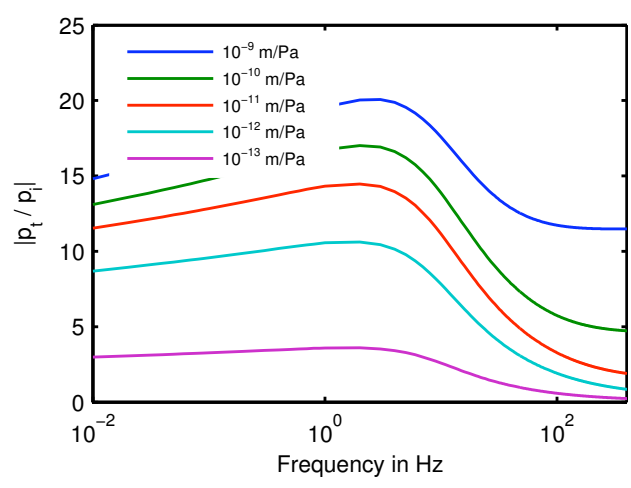

Figure 3: Tube to $\mathrm{P}$-wave pressure amplitude ratios plotted against frequency for different fracture compliance values. The parameters for this study are same as Figure 2.

0.1-1 mm and compliance from $10^{-14}-10^{-5} \mathrm{~m} / \mathrm{Pa}$ and plotted contours for constant values of amplitude ratio at a given frequency (see Figure 4). We can see that the amplitude ratio flattens after some aperture value (when $L_{0}>>\delta$ ) and is not greatly influenced by the aperture beyond certain point. At these large apertures, an observed amplitude ratio corresponds to a single compliance value. However, at smaller apertures, the contours bend towards higher compliance values. To retain the same amplitude ratio at smaller apertures, the fracture should be more compliant. So, every amplitude ratio corresponds to a minimum compliance. Thus, an amplitude ratio observed at an arbitrary frequency allows us to place a lower bound on the compliance. In general, we have data spanning a range of frequencies. So, we can set up an inverse problem to estimate the aperture and compliance such that the observed amplitudes at all the frequencies are satisfied within permissible error. However, we observe that the amplitude contours in compliance-aperture parameter space for frequencies in the high frequency regime are similar and the amplitude ratios are constant over those frequencies. That is, data collected in the high frequency regime do not give us additional information to constrain the compliance and aperture values, though it puts a bound on compliance values. It would be best to collect data spanning the transition regime to better constrain these parameters. For practical purposes, this is a frequency band of 1-50 $\mathrm{Hz}$. If a particular situation permits us to collect data with different fluids (say oil and water or different saturation levels of a multiphase fluid), we have additional constraints and a robust estimate.

We compared the amplitude ratios from Hardin et al. (1987) with the predictions from our model. Hardin et al. (1987) presented the data from a field experiment at a well in Mirror Lake, New Hampshire. The Mirror Lake borehole was drilled to a depth of about $225 \mathrm{~m}$ with a diameter of $0.15 \mathrm{~m}$ in a metamorphic sequence of schist and gneiss, intruded by thick, irregular veins of quartz monzonite. Standard wireline logs, FWAL (2-20 kHz) and hydrophone VSP (10-1000 Hz) data were collected. The VSP data were bandpass filtered and tubewave to $\mathrm{P}$-wave amplitude ratios were estimated over the frequency range $100-300 \mathrm{~Hz}$. The amplitude ratios, at a fre- 
Fracture compliance from tubewaves

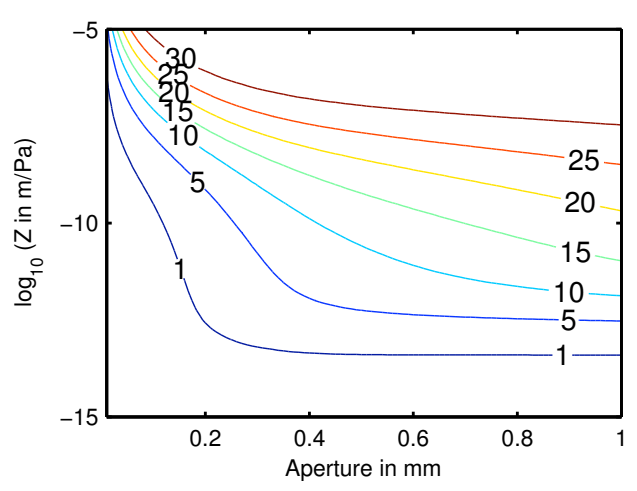

Figure 4: Amplitude ratio contours are plotted in the aperture, compliance parameter space for a frequency of $10 \mathrm{~Hz}$. The parameters for the study are same as Figure 2.

quency of frequency of $150 \mathrm{~Hz}$ (source band center frequency), corresponding to a fracture at $45 \mathrm{~m}$ depth, ranged from 10 to 15. Comparing these amplitude ratios to amplitude ratio contours (at $150 \mathrm{~Hz}$ ) from a model with parameters representing the field study, the lower bound on compliance was found to lie between $3 \times 10^{-10}-10^{-9} \mathrm{~m} / \mathrm{Pa}$ (see Figure 5). However, analyzing the same data, Hardin (1986) suggested that compliance values should be of the order of $10^{-12}-10^{-13} \mathrm{~m} / \mathrm{Pa}$. Hardin applied a model valid for the low frequency regime to data from the high frequency regime and thus under-estimated the fracture compliance. Similarly, we analyzed amplitude ratios corresponding to a fracture at $290 \mathrm{~m}$ in a water well at Hamilton, MA, drilled in a gabbro-granodiorite formation (Hardin, 1986). The lower bound on compliance for this fracture varied between $10^{-10}-10^{-9} \mathrm{~m} / \mathrm{Pa}$.

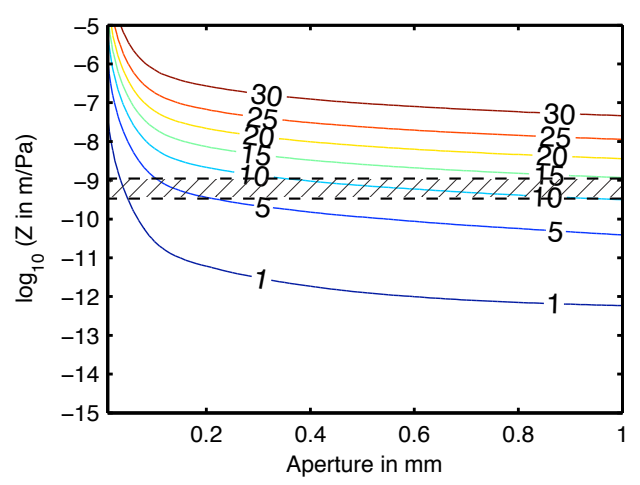

Figure 5: Amplitude ratio contours are plotted in the aperture, compliance parameter space for a frequency of $150 \mathrm{~Hz}$. Observed amplitude ratios between 10 and 15 suggest that the lower bound on the compliance lies between $3 \times 10^{-10} \mathrm{~m} / \mathrm{Pa}$ and $10^{-9} \mathrm{~m} / \mathrm{Pa}$. The parameters for the study are same as Figure 2 and correspond to the field study at Mirror Lake borehole.

\section{DISCUSSION}

The present model is developed for normal incidence on a fracture that intercepts the borehole at a right angle, e.g. vertical borehole and horizontal fracture. However, with increasing depth fractures are more inclined and tend to be sub-vertical. Moreover, the incident wavefield may not be parallel to the borehole. When a wave is incident at an angle to the borehole, the pressure amplitude of that wave measured in the borehole increases compared to that of a wave propagating parallel to the borehole (White, 1983). At the same time, non-normal incidence on a fracture reduces the normal stress on the fracture and decreases the pressure amplitude of the generated tubewave in the borehole. As a result, any deviation from the assumed geometry in the field would mean reduced amplitude ratios. In other words, inclined fractures should be more compliant than a horizontal fracture that generates the same amplitude ratio. In addition, the effect of asperities and tortuosity on flow would be to reduce the pressure amplitude ratios. So, the bound on compliance from our model is conservative.

Higher compliance values at larger scales is not surprising. Previously, Brown and Scholz (1985) analyzed the natural rock surfaces and found that the profiles are fractal and the spatial frequencies have a red-noise power spectra. Other authors (Hakami and Larsson, 1996) reported that apertures are normally distributed. This means that larger apertures are few and spread out spatially at larger distances. When a wave is incident on a fracture, it samples regions on the order of its wavelength. Larger wavelengths sample larger fracture surface and sample larger apertures. A few large apertures can drastically increase the compliance and increase fluid transmissivity. However, Brown and Scholz (1985) also suggest that fracture surfaces should be correlated after a certain scale. In such case, we will see increase in compliance with scale until we reach the correlation length.

Though we predict high compliance values (of the order of $10^{-10} \mathrm{~m} / \mathrm{Pa}$ ) for dry fractures, one should be careful when applying them to linear-slip model. A fluid filled fracture has a compliance similar to a dry fracture under drained conditions and becomes much stiffer due to the incompressibility of the fluid under un-drained conditions. The overall compliance could be frequency dependent, as a result of fluid motion, depending on the drainage length compared to the wavelength.

\section{CONCLUSIONS}

Tubewave generation at a fracture in a borehole is modeled accounting for the intrinsic fracture stiffness. The pressure field in the fracture was solved without any low/high approximations on frequency. Thus, amplitude ratios over a range of frequencies and fracture compliances were analyzed. Amplitude ratios peak near transition frequencies and increase with increasing compliance. It is observed that measurements taken near the transition frequency can constrain compliance and aperture better. Comparing the present model to a previously published VSP data suggested that fracture compliance of the order of $10^{-10}-10^{-9} \mathrm{~m} / \mathrm{Pa}$ can be expected in the field. With these values, scattering of seismic waves from such fractured regions should be observed using surface seismic data. 


\section{Fracture compliance from tubewaves}

\section{REFERENCES}

Brown, S. R., and C. H. Scholz, 1985, Broad bandwidth study of the topography of natural rock surfaces: Journal of Geophysical Research, 90, 12,575-12,582.

Burns, D. R., M. E. Willis, M. N. Toksoz, and L. Vetri, 2007, Fracture properties from seismic scattering: The Leading Edge, 26, 1186-1196.

Grandi, S., 2008, Multiscale determination of in situ stress and fracture properties in reservoirs: Ph.D thesis, MIT.

Hakami, E., and E. Larsson, 1996, Aperture measurements and flow experiments on a single natural fracture: International Journal of Rock Mechanics and Mining Sciences \& Geomechanics Abstracts, 33, 395-404.

Hardin, E., 1986, Fracture characterization from attenuation and generation of tubewaves: M.S. thesis, MIT, Cambridge.

Hardin, E. L., C. H. Cheng, F. L. Paillet, and J. D. Mendelson, 1987, Fracture characterization by means of attenuation and generation of tube waves in fractured crystalline rock at Mirror lake, New Hampshire: Journal of Geophysical Research, 92 , 7989-8006.

Ionov, A. M., 2007, Stoneley wave generation by an incident p-wave propagating in the surrounding formation across a horizontal fluid-filled fracture: Geophysical Prospecting, 55, 71-82.

Kachanov, M., 1992, Effective elastic properties of cracked solids: Critical review of some basic concepts: Applied Mechanics Reviews, 45, 304-335.

King, M., L. Myer, and J. Rezowalli, 1986, Experimental studies of elastic-wave propagation in a columnar-jointed rock mass: Geophysical Prospecting, 34, 1185-1199.

Lee, M. W., and A. H. Balch, 1982, Theoretical seismic wave radiation from a fluid-filled borehole: Geophysics, 47, 1308-1314.

Lubbe, R., J. Sothcott, M. H. Worthington, and C. McCann, 2008, Laboratory estimates of normal and shear fracture compliance: Geophysical Prospecting, 56, 239-247.

Lubbe, R., and M. H. Worthington, 2006, A field investigation of fracture compliance: Geophysical Prospecting, 54, 319-331.

Peacock, S., and J. A. Hudson, 1990, Seismic properties of rocks with distributions of small cracks: Geophysical Journal International, 102, 471-484.

Pyrak-Nolte, L. J., L. R. Myer, and N. G. W. Cook, 1990, Transmission of seismic waves across single natural fractures: Journal of Geophysical Research, 95, 8617-8638.

Schoenberg, M., 1980, Elastic wave behavior across linear slip interfaces: The Journal of the Acoustical Society of America, 68, $1516-1521$.

Tang, X., 1990, Acoustic logging in fractured and porous formations: Ph.D thesis, MIT, Cambridge.

White, J., 1983, Underground sound: Application of seismic waves: Elsevier science publishing company.

Willis, M. E., D. R. Burns, R. Rao, B. Minsley, M. N. Toksoz, and L. Vetri, 2006, Spatial orientation and distribution of reservoir fractures from scattered seismic energy: Geophysics, 71, O43-O51.

Worthington, M. H., and J. A. Hudson, 2000, Fault properties from seismic Q: Geophysical Journal International, 143, 937-944.

Worthington, M. H., and R. Lubbe, 2007, The scaling of fracture compliance: Geological Society, London, Special Publications, $270,73-82$. 